\title{
APLICACIÓN DE ECUACIONES DE CONICIDAD PARA TECA (Tectona grandis L.F.) EN LA ZONA COSTERA ECUATORIANA
}

\author{
${ }^{\bullet}$ Carlos Esteban Lara Vásquez ${ }^{1}$ \\ ${ }^{I}$ Departamento de Ciencias Forestales. Universidad Nacional de Colombia, Sede Medellín. Calle 59A 63 20. Bl. 20 Of. 215. \\ Medellín,Colombia.`celarav@unal.edu.co
}

Resumen

$\mathrm{S}_{\mathrm{c}}^{\mathrm{e}}$ modelaron cinco funciones no segmentadas de conicidad para Tectona grandis, especie forestal que ha incrementado notoriamente su área plantada en la zona costera ecuatoriana y donde se desconocen formulaciones de este tipo. Se realizó un proceso de regresión no lineal por el método de máxima verosimilitud a una muestra de 125 árboles y se evaluó su ajuste. Posteriormente, se hizo un proceso de validación independiente, se aplicaron pruebas no paramétricas y un análisis por altura relativa, para identificar fortalezas y debilidades en cada parte del fuste. La función polinomial de quinto grado presentó el mejor ajuste (Sesgo Absoluto $=1.009$, Índice de Ajuste $=0.962$ ), por ello se calculó la forma integrada de esta ecuación. Se espera que estos modelos desarrollados y sus bondades de aplicación puedan ser de utilidad para la comunidad forestal ecuatoriana.

Palabras claves: conicidad, Ecuador, manejo forestal, teca, Tectona grandis.

\section{Abstract}

Zive non-segmented taper functions were modeled for 1 Tectona grandis, which has increased significantly their planted area in Coastal Ecuador were formulations of this type are unknown. A process of nonlinear regression (maximum likelihood) was performed to a sample of 125 trees with fit evaluation. Subsequently, an independent validation process were performed, non parametric tests and a relative height analysis in order to identify strengths and weaknesses in each part of the shank was also applied. The fifth grade polynomial function showed the best fit (Absolute Bias $=1.009$, Fit Index $=0.962$ ), for that reason, the integrated form of this equation was calculated. It is expected that these developed models and their application benefits could be useful for the forestry comunity in Ecuador.

Key words: taper equations, Ecuador, forest management, teak, Tectona grandis.

\section{INTRODUCCIÓN}

$\mathrm{D}^{2}$ urante las últimas décadas, en la zona costera ecuatoriana se ha incrementado notoriamente el área reforestada con teca (Tectona grandis), lo cual puede fortalecer el desarrollo forestal del país, debido a esto, es indispensable generar herramientas propias que permitan mejorar el manejo de los rodales con el fin de obtener mayor productividad; algunos esfuerzos se conocen al respecto (Crespo et al., 2008; Suatunce et al., 2010; Flores et al., 2010). Sin embargo, hasta ahora no se han publicado ecuaciones de volumen o conicidad para teca en Ecuador, las cuales son el insumo principal para conocer las existencias de los rodales, por lo cual este trabajo se constituye en una herramienta para favorecer la toma de decisiones en las plantaciones forestales.

Típicamente, el volumen total de un árbol se estima a partir de ecuaciones de volumen, lo cual es útil y práctico; sin embargo, este tipo de formulaciones sólo permiten conocer el volumen total del árbol (Prodan et al., 1997), lo que resulta en una desventaja a la hora de analizar e interpretar parámetros de evaluación dasométricos. Por otra parte, se han propuesto ecuaciones de ahusamiento o conicidad, que permiten conocer el diámetro en cualquier altura del árbol y viceversa (Clutter et al., 1983). Estas funciones tienen la gran bondad de permitir la obtención del volumen para cualquier diámetro por medio de su integración matemática (Biging, 1984), esto es útil para detallar las existencias de madera en los rodales. Es por esto que este estudio tuvo como objetivo modelar y validar de manera independiente ecuaciones de conicidad con información propia de la zona costera ecuatoriana, además de integrar matemáticamente el mejor modelo obtenido.

Recibido: 31-Octubre-2011. Recibido en forma corregida: 22-Diciembre 


\section{MÉtodos}

\section{Área de estudio}

Z ste trabajo se desarrolló en plantaciones forestales Ede teca (Tectona grandis) pertenecientes a la empresa Tropibosques S.A., proyecto localizado en la parte centro-occidental de Ecuador, cantón Balzar, provincia del Guayas. El sitio pertenece al bosque seco Tropical (Holdridge, 1978) y presenta un régimen de precipitación unimodal con un período lluvioso en el primer trimestre del año y una marcada sequía durante el resto del año, la precipitación promedia reportada fue $1,222 \mathrm{~mm}$, la temperatura media anual de $24.4^{\circ} \mathrm{C}$ y la humedad relativa de $72.9 \%$ (Flores et al., 2010). La altitud varía entre 40 a $80 \mathrm{~m}$ y el relieve presenta una forma ondulado-colinado (Tropibosques, 2007). Los suelos son profundos, drenados y se caracterizan por contenidos de materia orgánica de medios a bajos y ligeramente ácidos (Tropibosques, 2007), de textura arcillo-arenosa (Flores et al., 2010).

\section{Ajuste y validación de los modelos}

La muestra estuvo compuesta por 125 árboles con diámetros entre $[$ mín. $=1.8 \mathrm{~cm}$, máx. $=40.8 \mathrm{~cm}$ ] y alturas entre $[$ mín. $=2.1 \mathrm{~m}$, máx. $=22.7 \mathrm{~m}], 100 \mathrm{de}$ ellos se emplearon para la construcción de los modelos y 25 para el proceso de validación independiente. En campo se seleccionaron los individuos al azar, fueron apeados desde la base y trozados cada $2 \mathrm{~m}$, se midió el diámetro inferior, medio y superior de cada troza y la longitud total se obtuvo como la suma de las secciones. Se modelaron cinco funciones de conicidad no segmentadas (Cuadro 1), propuestas por: Ormerod (1973), Borges (1981), González (1988), Kozak (1988) y Figueiredo et al. (1996), denominado en adelante polinomial y Forslund (1990).

\section{Cuadro 1. Funciones no segmentadas de conicidad evaluadas en este estudio}

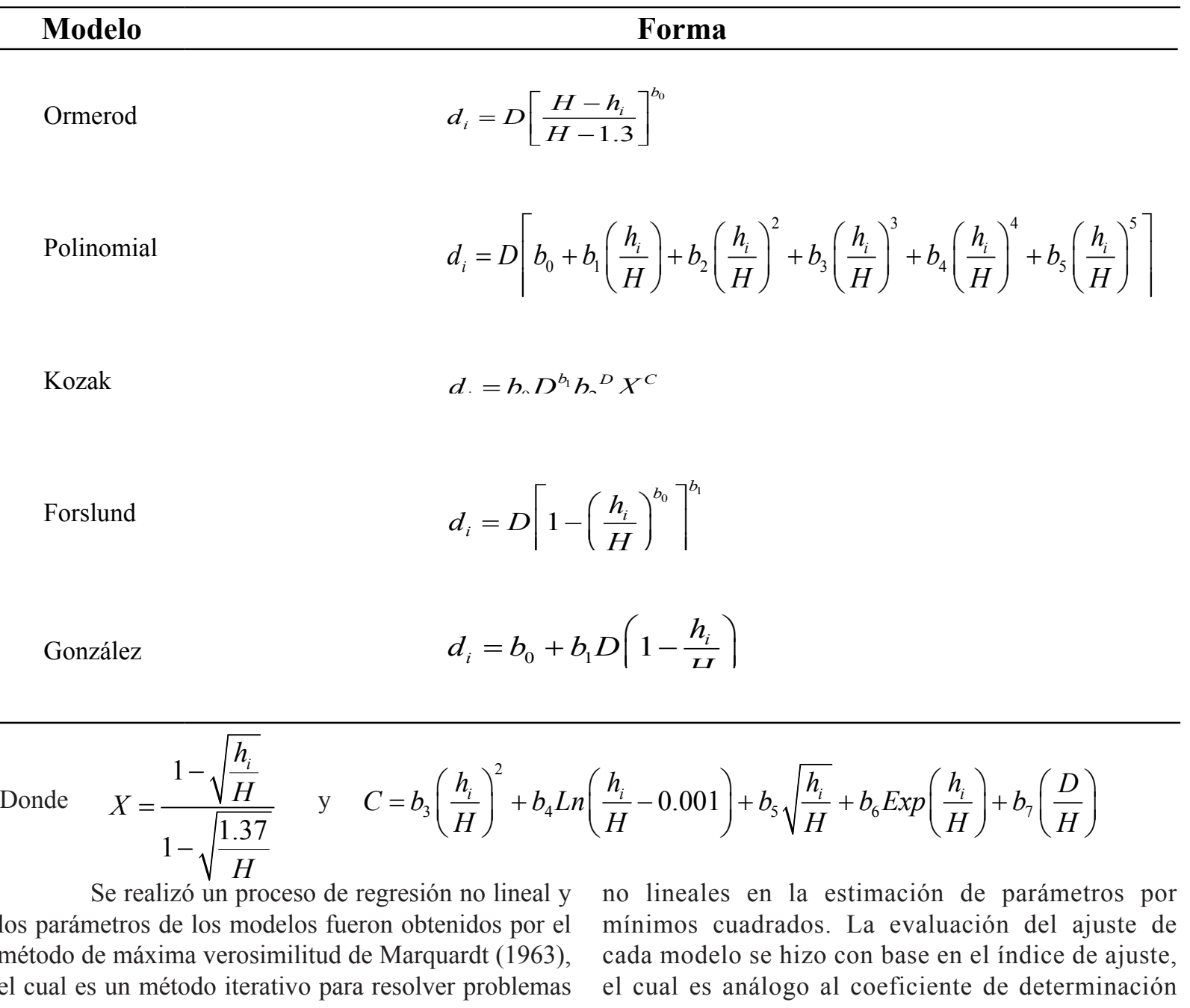


ajustado (Ecuación 1), el valor absoluto del sesgo (Ecuación 2) y el error estándar de los estimados (Ecuación 3) (Schlaegel, 1981; Jiang et al., 2005; Brooks et al., 2008). Se evaluó la autocorrelación de errores por medio del índice Durbin-Watson (DW) y el comportamiento gráfico de los residuales estandarizados. Adicionalmente, se realizó un proceso de validación independiente (con una muestra no empleada en la construcción de los modelos) con base en dos pruebas no paramétricas: el test de KolmogorovSmirnov (KS) y el test Wilcoxon (Wil). Por último, se evalúo el comportamiento del sesgo absoluto por alturas relativas (Garber y Maguire, 2003; Brooks et al., 2008), para valorar el ajuste del modelo en cada parte del fuste.

$$
I A=1-\left[\frac{\sum_{i-1}^{n}\left(Y_{i}-\hat{Y}_{i}\right)^{2}}{\sum_{i-1}^{n}\left(Y_{i}-\bar{Y}_{i}\right)^{2}}\right] \quad[1] \quad S E S G O=\frac{\sum_{i-1}^{n}\left(Y_{i}-\hat{Y}_{i}\right)}{\mathrm{n}} \quad \text { [2] } \quad S E E=\sqrt{\frac{\sum_{i-1}^{n}\left(Y_{i}-\hat{Y}_{i}\right)^{2}}{n-k}}
$$

Donde: $\quad Y_{i}$ es el valor observado, $\hat{Y}_{i}$ es el valor estimado, $\bar{Y}_{i}$ es el valor promedio estimado y $n$ es el número de observaciones.

\section{Resultados y Discusión}

\section{Evaluación de los modelos}

口n general, las cinco funciones de conicidad Epresentaron un bajo sesgo $<1.3 \mathrm{~cm}$, un bajo error estándar de los estimados y un alto índice de ajuste (Cuadro 2). La función polinomial presentó el mejor ajuste. El índice de Durbin-Watson en todos los casos fue $>0.8$ lo cual indica presencia de autocorrelación de errores inevitable en modelos de este tipo, pero sin incidencia a la hora de las estimaciones. Por su parte, en el cuadro 3 se presentan los coeficientes de regresión obtenidos para cada modelo, los cuales permiten usar cada ecuación de conicidad.

Cuadro 2. Indicadores estadísticos básicos obtenidos para cada función de conicidad. $\mathrm{SA}=$ sesgo absoluto, SEE = error estándar de los estimados, IA = índice de ajuste, DW: estadístico Durbin-Watson

\begin{tabular}{lcccc}
\hline \multicolumn{1}{c}{ Modelo } & SA $(\mathbf{c m})$ & SEE $(\mathbf{c m})$ & IA & DW \\
\hline Ormerod & 1.057 & 1.706 & 0.945 & 0.929 \\
Polinomial & 1.009 & 1.409 & 0.962 & 0.852 \\
Kozak & 1.087 & 0.806 & 0.937 & 1.059 \\
Forslund & 1.285 & 1.973 & 0.927 & 0.911 \\
González & 0.954 & 1.543 & 0.955 & 1.157 \\
\hline
\end{tabular}

Cuadro 3. Coeficientes de regresión obtenidos para cada función de conicidad

\begin{tabular}{cccccc}
\hline Parámetro & Ormerod & Forslund & González & Polinomial & Kozak \\
\hline $\boldsymbol{b}_{0}$ & 0.85678 & 1.16238 & 1.48869 & 1.30325 & 0.35821 \\
$\boldsymbol{b}_{1}$ & & 0.86101 & 1.03853 & -5.05635 & 0.81557 \\
$\boldsymbol{b}_{2}$ & & & 20.38000 & 0.00296 \\
$\boldsymbol{b}_{3}$ & & & -41.75950 & 6.62045 \\
$\boldsymbol{b}_{4}$ & & & 37.62180 & -1.51848 \\
$\boldsymbol{b}_{5}$ & & & -12.39520 & 14.59910 \\
$\boldsymbol{b}_{6}$ & & & & -7.70550 \\
$\boldsymbol{b}_{7}$ & & & & & 0.10480 \\
\hline
\end{tabular}


Cada modelo presentó su propio comportamiento (Figura 1) y los modelos denominados Ormerod, Forslund, Kozak y González tienden a presentar un desajuste cuando se alcanzan valores altos de diámetro (Figura 1), esta tendencia es más notoria en el comportamiento de los residuales estandarizados (Figura 2) y estos modelos mencionados presentan
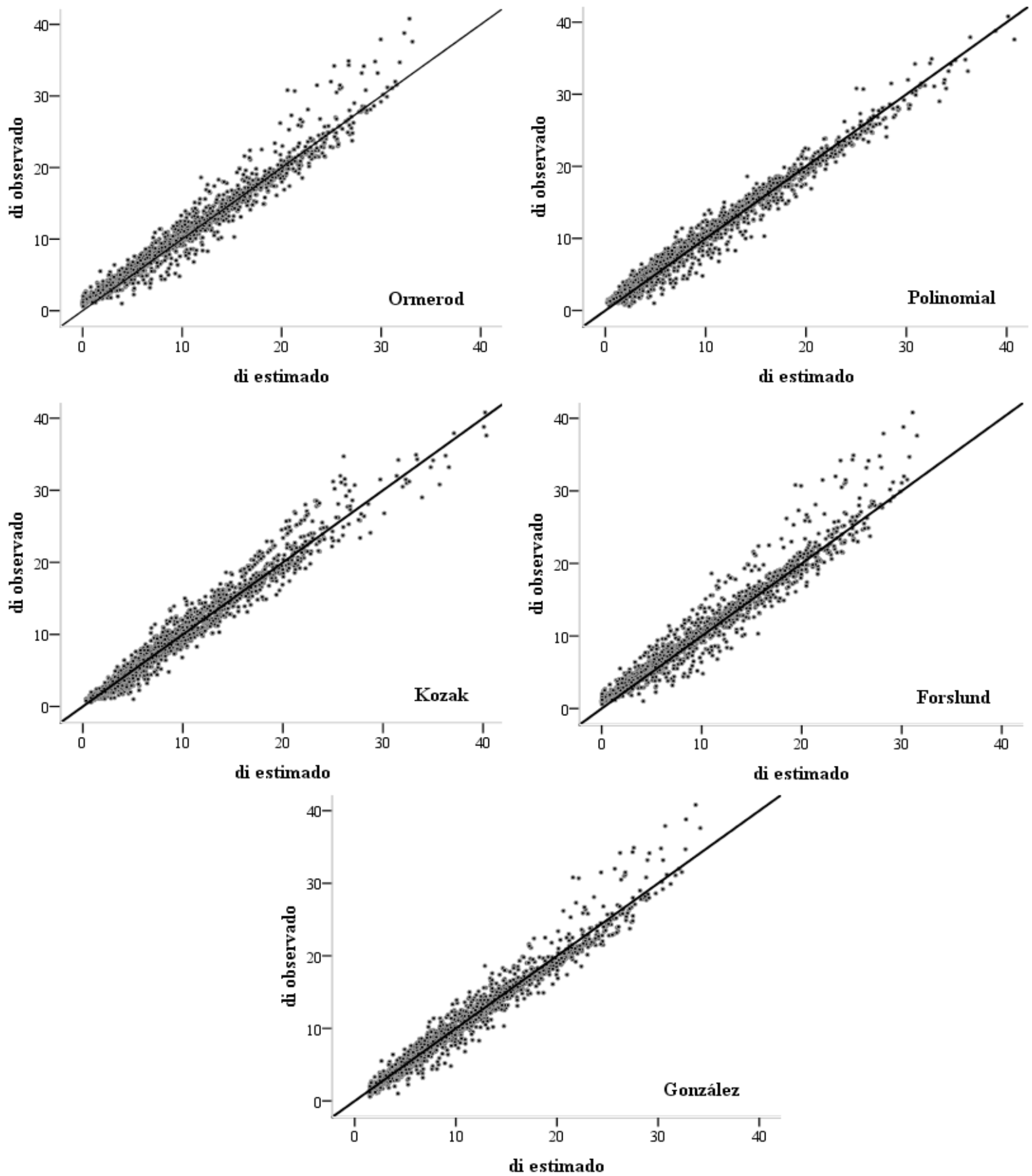

Figura 1. Comportamiento gráfico de cada función de conicidad evaluada. Diámetro observado y estimado en centímetros. 


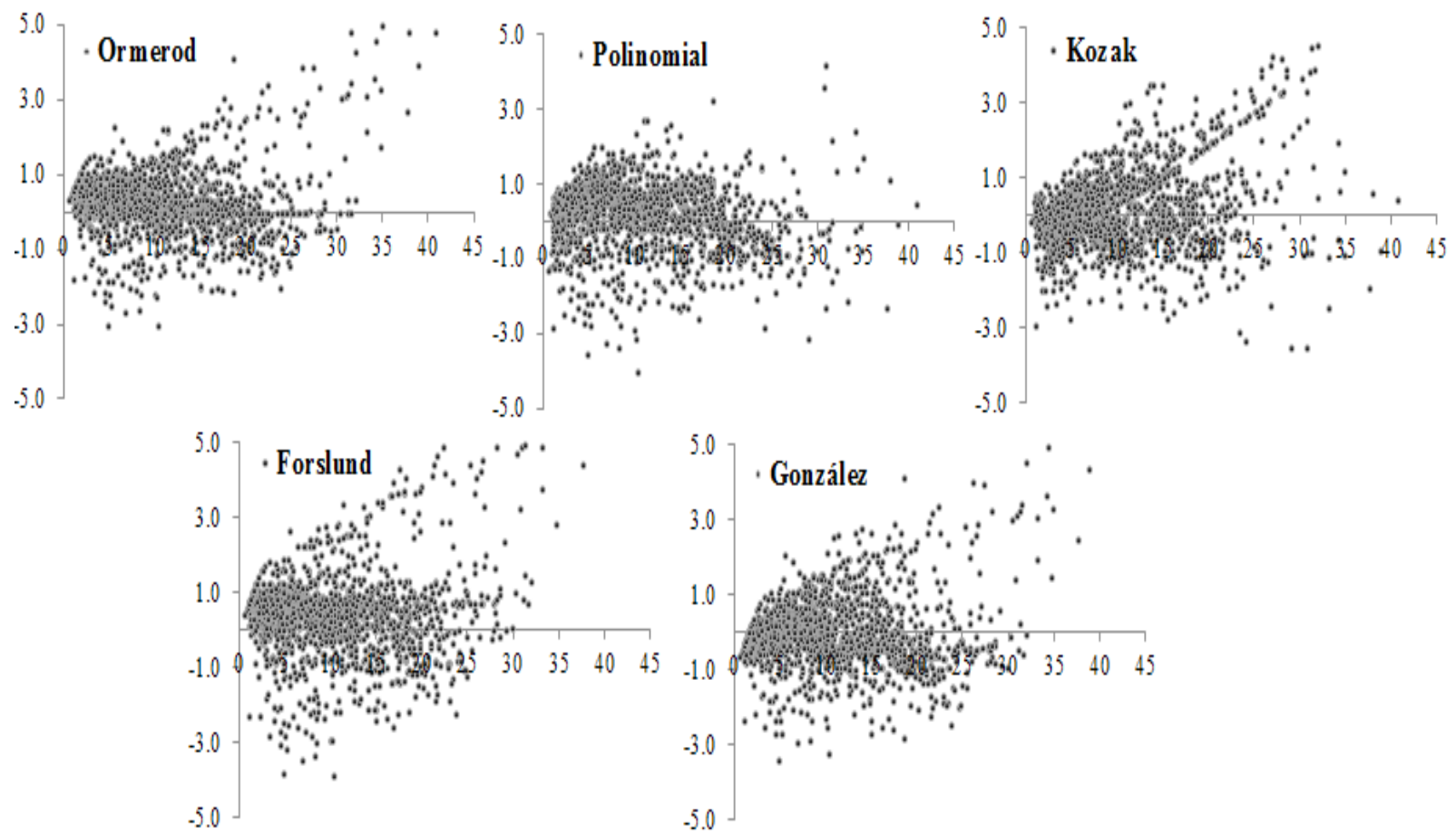

Figura 2. Comportamiento de los residuales estandarizados, en el eje de abscisas se encuentra el diámetro normal $(\mathrm{cm})$ y en el eje de ordenadas los valores de los residuales estandarizados para cada par de datos empleados en la construcción de los modelos.

Validación de los modelos

Los resultados de la validación independiente se resumen en el cuadro 4, se puede notar que el modelo polinomial obtuvo el menor valor de sesgo absoluto y el índice de ajuste más alto. En todos los casos las pruebas no paramétricas presentaron $p$-values superiores a 0.05 , lo cual indica la inexistencia de diferencias significativas entre los diámetros observados y los estimados.

En la evaluación del sesgo absoluto por alturas relativas se observa una tendencia a presentar desajustes al principio y al final del fuste en todos los modelos (Figura 3), es decir, que la predicción en la primera parte del fuste es poco precisa, debido a que existe una variación alta; no obstante, todas las funciones presentan en la mayoría de casos valores predichos por debajo de $1.0 \mathrm{~cm}$ de sesgo absoluto (Figura 3), incluso el modelo González (1988) al ser evaluado por alturas relativas mostró que tiene un comportamiento consistente ya que en todas las clases de altura su sesgo absoluto fue $<1$ $\mathrm{cm}$ y debido a que este modelo tiene una formulación matemática simple, podría también ser implementado para la estimación de los diámetros en cualquier altura del fuste; no obstante, debe considerarse que este modelo presentó heterocedasticidad.

Cuadro 4. Resultados de los diferentes indicadores estadísticos en el proceso de validación independiente. SA = sesgo absoluto, IA = índice de ajuste, KS: Kolmogorov-Smirnov y Wil = Wilcoxon. Todos los test con un nivel de significancia del $95 \%$

\begin{tabular}{lcccc}
\hline \multicolumn{1}{c}{ Modelo } & SA (cm) & IA & KS (valor-p) & Wil (valor-p) \\
\hline Ormerod & 0.895 & 0.945 & 0.468 & 0.208 \\
Polinomial & 0.783 & 0.969 & 0.406 & 0.164 \\
Kozak & 0.886 & 0.958 & 0.958 & 0.977 \\
Forslund & 1.081 & 0.921 & 0.251 & 0.670 \\
González & 0.807 & 0.960 & 0.958 & 0.998 \\
\hline
\end{tabular}



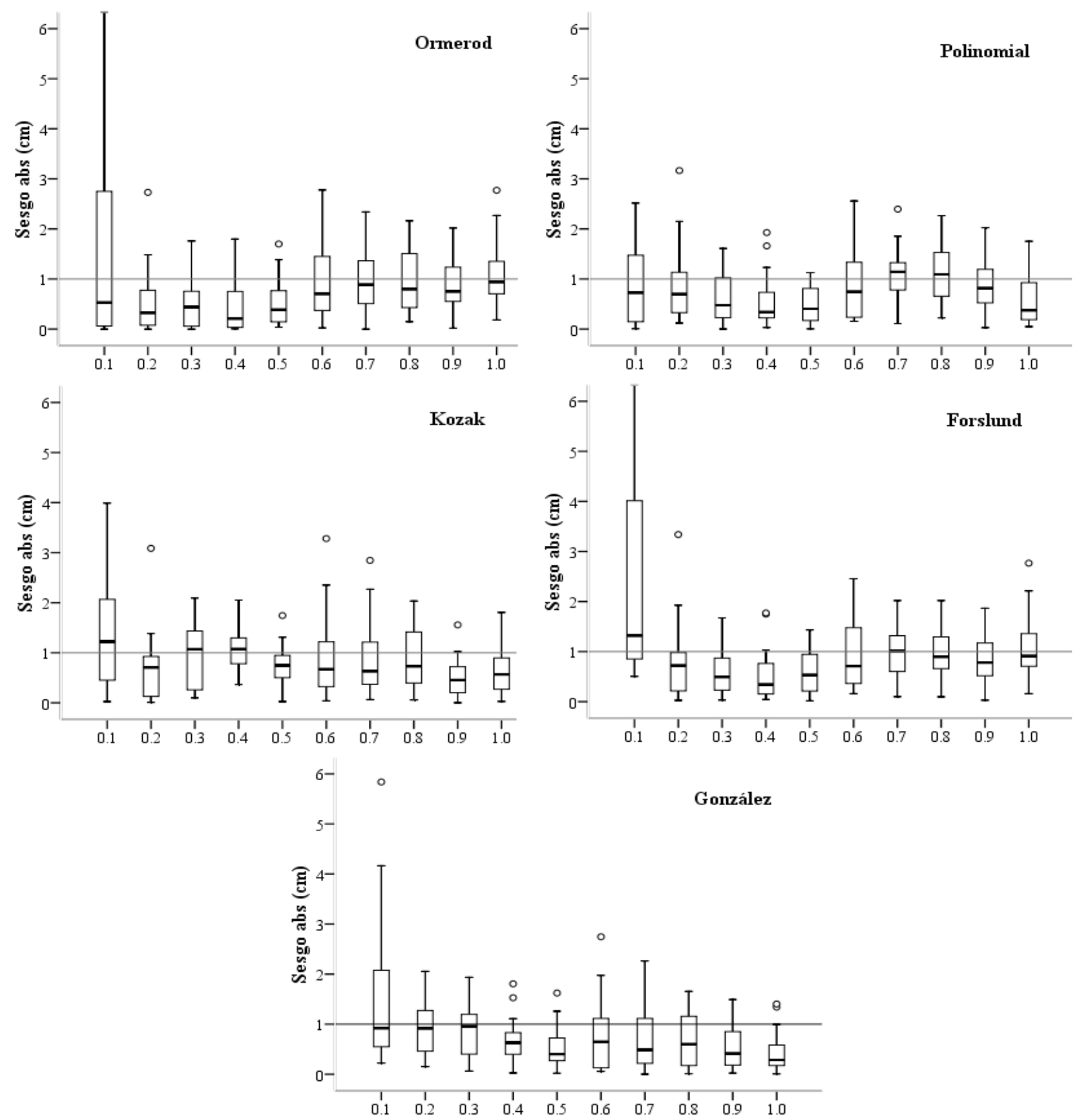

Figura 3. Box-plot que describe el comportamiento del sesgo absoluto en las diferentes alturas relativas del fuste para cada función de conicidad, en el eje de absisas se encuentran los rangos de altura relativa entre 0.1 y 1.0. La línea horizontal gris delimita el sesgo absoluto en $1 \mathrm{~cm}$.

En Venezuela, De Pinto et al. (2003) desarrollaron modelos de conicidad de exponente variable para teca de tipo Kozak (1988) y Pérez et al. (1990), obteniéndose buen ajuste; sin embargo, ellos no evaluaron otro tipo de modelos; en este trabajo se encontró que los modelos de exponente variable presentaron deficiencias en las estimaciones del diámetro en la parte inicial y final del fuste y aunque fueron estimados por el método de máxima verosimilitud es notoria la anormalidad en el comportamiento de los residuales estandarizados.

Por su parte, en Brasil, Figueiredo et al. (2006) ajustaron tres tipos de modelos polinomiales para teca y encontraron buenos resultados, aunque el modelo polinomial de quinto grado tuvo el tercer puesto en esa investigación ya que presentó una sobreestimación en la parte final del fuste, lo cual no ocurrió para nuestro estudio, debido a que de los cinco modelos evaluados, el polinomial presentó el menor sesgo en la parte final del fuste; no obstante, sería interesante evaluar el modelo 
polinomial propuesto por Goulding y Murray (1976), dado que presentó muy buen ajuste para los árboles de teca brasileros.

Con respecto al modelo potencial de Forslund (1990) y sigmoideo de Ormerod (1973), no se conoce que hayan sido evaluados en teca; sin embargo, Dorado y Álvarez (2000) encontraron en Pinus radiata que estos modelos son débiles frente a modelos de exponente variable, aunque en ese caso no ajustaron modelos polinomicos. Otro tipo de modelos de conicidad trigonométricos (Thomas y Parresol, 1991; Bi, 2000) y segmentados (Max y Burkhart, 1976) hasta ahora no han sido ajustados para teca, pero han presentado buenas predicciones en otras especies forestales, por lo cual sería interesante desarrollarlos para conocer su ajuste con la conicidad que presenta los árboles de teca en Ecuador.
Volumen

Previo a este trabajo, Lara (2007) propuso una ecuación de volumen para $T$. grandis (Ecuación 4) con la misma base de información del presente estudio, esta función permite obtener el volumen total de cada árbol de manera directa y a partir de ella se podría construir una tabla de volumen. Sin embargo, esto limita el conocimiento detallado sobre las existencias de madera en los rodales y la posibilidad de estimar el volumen a partir de diámetros mínimos de corta preestablecidos. Es por esto que en este estudio se resalta la utilidad de las ecuaciones de conicidad en su forma integrada para lograr más precisión en la estimación. Por ello, el mejor modelo ajustado (polinomial) fue integrado matemáticamente (Ecuaciones: 5, 6, 7) y se puede usar para calcular el volumen en cualquier altura del fuste en cualquier individuo.

$$
\begin{gathered}
V=0.00017023(D)^{1.7315}(H)^{0.719286} \\
V=\frac{\pi}{40,000} \int_{h_{1}}^{h_{2}} d^{2} \partial h \\
\left(\frac{d_{i}}{D}\right)^{2}=\left[b_{0}+b_{1}\left(\frac{h_{i}}{H}\right)+b_{2}\left(\frac{h_{i}}{H}\right)^{2}+b_{3}\left(\frac{h_{i}}{H}\right)^{3}+b_{4}\left(\frac{h_{i}}{H}\right)^{4}+b_{5}\left(\frac{h_{i}}{H}\right)^{5}\right]^{2}
\end{gathered}
$$

Luego la forma integrada sería:

$$
V=\frac{\pi D^{2}}{40,000}\left(\frac{1}{H^{10}}\left(\begin{array}{l}
b_{1}^{2} H^{10} h_{i}+\left(\frac{2 b_{1} b_{3}+b_{2}^{2}}{3}\right) H^{8} h_{i}^{3}+\left(\frac{2 b_{1} b_{5}+2 b_{2} b_{4}+b_{3}^{2}}{5}\right) H^{6} h_{i}^{5} \\
+\left(\frac{b_{1} b_{6}+b_{2} b_{5}+b_{3} b_{4}}{3}\right) H^{5} h_{i}^{6}+\left(\frac{b_{1} b_{4}+b_{2} b_{3}}{2}\right) H^{7} h_{i}^{4}+b_{1} b_{2} H^{9} h_{i}^{2} \\
+\left(\frac{2 b_{2} b_{6}+2 b_{3} b_{5}+b_{4}^{2}}{7}\right) H^{4} h_{i}^{7}+\left(\frac{b_{3} b_{6}+b_{4} b_{5}}{4}\right) H^{3} h_{i}^{8} \\
+\left(\frac{2 b_{4} b_{6}+b_{5}^{2}}{9}\right) H^{2} h_{i}^{9}+\left(\frac{b_{5} b_{6}}{5}\right) H h_{i}^{10}+\left(\frac{b_{6}^{2}}{11}\right) h_{i}^{11}
\end{array}\right) \|_{h_{1}}\right.
$$

Donde: $V$ : volumen en metros cúbicos, $D$ : diámetro normal en centímetros $(1.3 \mathrm{~m}), H$ : altura total en metros, $d i$ : diámetro en el punto $i, h i$ : altura en el punto $i$.

\section{Conclusiones}

T a elección de un modelo de conicidad debe considerar, además de los estadísticos tradicionales, el comportamiento en las diferentes partes del fuste.
En este caso, el modelo polinomial resultó robusto estadísticamente y además es consistente en todas las alturas relativas del fuste. Sin embargo, a futuro se 
debe implementar modelos polinomiales segmentados y trigonométricos para determinar la capacidad de clasificar mejor la forma de la teca en Ecuador, en especial, verificando las diferencias en la primera y última parte del fuste. Se espera que las formulaciones matemáticas propuestas en este trabajo, sirvan como base para ampliar las posibilidades de precisión de las existencias de madera en los rodales de teca en Ecuador de acuerdo con clasificaciones de productos a partir de diámetros de troza predeterminados. Es factible complementar nuestra base de datos con información de otras plantaciones forestales de teca de la zona, para recalcular los modelos buscando integrar toda la variación existente en la forma de los árboles de teca en la zona costera ecuatoriana.

Finalmente, se debe resaltar que durante varias décadas se han realizado modelos de conicidad; sin embargo, aún es necesario realizar más formulaciones de este tipo, en particular para especies exóticas que se han convertido en la base del crecimiento forestal en países del neotrópico. Los esfuerzos más representativos se han centrado en algunas especies del genero Pinus en Brasil y México, pero en países como Colombia y Ecuador han sido poco estudiados, esto se hace más evidente en zonas bajas, donde especies como la teca tienen un impacto notable y aunque se han publicado ecuaciones de conicidad para teca en Brasil y Venezuela aún es necesario profundizar en el conocimiento sobre su biometría.

\section{LiTERATURA CITADA}

Bi, H. 2000. Trigonometric variable-form taper equations for Australian eucalyptus. For. Sci. 46: 397-409.

Biging, G. S. 1984. Taper Equations for Second-Growth Mixed Conifers of Northern California. Forest. Sci. 30 (4):1103-1117.

Borges, J. F. 1981. Seccionamento do fuste de Pinus taeda L. para obtencao do volume de madeira serrada através da funcao polinomial. Curitiba. Dissertacao (Maestrado em Ciencias Florestais) Setor de Ciencias Agrárias. Universidade Federal do Paraná.

Brooks, J. R., L. B. Jiang y R. Ozcelik. 2008. Compatible stem volume and taper equations for Brutian pine, Cedar of Lebanon, and Cilicica fir in Turkey. For. Ecol. Man. 256:147-151.

Clutter, J. L., J. C. Fortson, L. V. Pienaar, G. H. Brister y R. L. Bailey. 1983. Timber management: A quantitative approach. John Wiley \& Sons, Inc.

Crespo, R., E. Jiménez y P. Suatunce. 2008. Análisis comparativo de las propiedades físico-mecánicas de la madera de Teca (Tectona grandis L. F.) de
Quevedo y Balzar. Ciencia y Tecnología 1: 55-63.

De Pinto, M., E. Marina y A. Mora. 2003. Evaluación de curvas de perfil para Tectona grandis L. con errores correlacionados en la Unidad Experimental Reserva Forestal de Caparo Venezuela. Rev. For. Ven. 47 (1):1-23.

Dorado, F. y J. G. Álvarez. 2000. Construcción de una tarifa de cubicación con clasificación de productos para Pinus radiata D. Don en Galicia basada en una función de perfil del tronco. Invest. Agr.: Sist. Recur. For. 9 (2):253-268.

Figueiredo, A., B. E. Borders y K. L. Hitch. 1996. Taper equations for Pinus taeda plantations in Southern Brazil For. Ecol. Man. 83:39-46.

Figueiredo, E. O., J. R. Scolforo y A. D. de Oliveira. 2006. Seleção de modelos polinomiais para representar o perfil e volumen do fuste de Tectona grandis L. F. Acta Amazónica 36 (4):465-482.

Flores, T., R. Crespo y F. Cabezas. 2010. Plagas y enfermedades en plantaciones de Teca (Tectona grandis L.F) en la zona de Balzar, provincia del Guayas. Ciencia y Tecnología 3(1):15-22.

Forslund, R. 1990. The power function as a simple stem profile examination tool. Can. J. For. Res. 21:193198.

Garber, S. M. y D. A. Maguire. 2003. Modeling stem taper of three central Oregon species using nolinear mixed effects models and autoregressive error structures. For. Ecol. Man. 179:507-522.

González, H. 1988. La conicidad de los árboles y algunas posibles aplicaciones en el manejo de bosques. Rev. Fac. Nal. Agr. 41 (1):51-60.

Goulding, C. J. y J. C. Murray. 1976. Polynomial taper equations that are compatible with tree volume equations. New Zealand Jor. For. Sci. 5(3):313322.

Holdrige, L. R. 1978. Ecología basada en zonas de vida. Instituto Interamericano de cooperación para la agricultura. San José, Costa Rica.

Jiang, L., J. R. Brooks y J. Wang. 2005. Compatible taper and volume equations for yellow-poplar in West Virginia. For. Ecol. Man. 213:399-409.

Kozak, A. 1988. A variable-exponent taper equation. Can. J. For. Res. 18:1363-1368.

Lara, C. E. 2007. Pasantía en plantaciones forestales de Tectona grandis Linn F. en Ecuador. Dep. Ciencias Forestales. Universidad Nacional de Colombia Sede Medellín.

Marquardt, W. D. 1963. An algorithm for least squares estimation of non linear parameters. Jour. Soc. Ind. App. Math. 11(2):431-444.

Max, T.A. y H. E. Burkhart. 1976. Segmented polynomial regression applied to taper equation. For. Sci. 22(33):283-289. 
Ormerod, D. W. 1973. A simple bole model. For. Chron. 49, 136-138.

Pérez, D., H. E. Burkhart y C. T. Stiff. 1990. A variableform taper function for Pinus oocarpa in Central Honduras. Forest Science. 36 (1):186-191.

Prodan, P., R. Peters, F. Cox y P. Real. 1997. Mensura Forestal. Instituto Interamericano de Cooperación para la Agricultura (ICCA). Deutsche Gesellschaft fur Technische Zusammenarbeit (GTZ). Costa Rica.

Schlaegel, B. E. 1981. Testing, reporting, and using biomass estimation models p. 95-112. In: Proceedings of the Southern Forest Biomass Workshop.
Suatunce, P., G. Díaz y L. García. 2010. Evaluación de la densidad de plantación en el crecimiento de cuatro especies forestales tropicales. Ciencia y Tecnología 3(1):23-26.

Thomas, C. E. y B. R. Parresol. 1991. Simple, flexible trigonometric taper equations. Can. J. For. Res. 21:1132-1137.

Tropibosques S.A. 2007. Plan de Manejo Ambiental. Reforestadora de Bosques Tropicales Tropibosques S.A. 